1972-02-01

\title{
Electron Cyclotron Radiation Measurements to Determine Relative Electron Temperatures in A Low-Density Magnetic-Mirror Plasma System
}

Andrew L. Gardner

Follow this and additional works at: https://scholarsarchive.byu.edu/facpub

Part of the Electrical and Computer Engineering Commons

\section{Original Publication Citation}

Gardner, Andrew L. "Electron Cyclotron Radiation Measurements to Determine Relative Electron Temperatures in A Low-Density Magnetic-Mirror Plasma System." Nuclear Science, IEEE Transactions on 19.1 (1972): 757-6

\section{BYU ScholarsArchive Citation}

Gardner, Andrew L., "Electron Cyclotron Radiation Measurements to Determine Relative Electron Temperatures in A Low-Density Magnetic-Mirror Plasma System" (1972). Faculty Publications. 791. https://scholarsarchive.byu.edu/facpub/791 accepted for inclusion in Faculty Publications by an authorized administrator of BYU ScholarsArchive. For more information, please contact ellen_amatangelo@byu.edu. 


\section{Andrew L. Gardner*}

Department of Physics and Astronony, Brigham Young University Provo, Utah 84601

\section{Summaxy}

Measurements of the electron cyclotron radiation emitted from the plasma of the Alice system? were made using a synchronous detection microwave radiometer in the range of $10.2-11.6 \mathrm{GHz}$. Both sidebands were used to sample radiation from two nested closed shells within the plasma. For a temous plasm this radiation is proportional to the product of the electron density and the electron temperature. Since measurements of the plasma density were available from other detectors, relative values of the electron temperature were obtained using the measured radiation intensity.

\section{The Plasma Configuration}

The experimental arrangement of the plasma system has been described previously. 1 The plasma is established and confined in a magnetic well produced by a current that travels in a path shaped like the seam of a baseball.

The current path and a portion of the surface of a magnetic flux tube are sketched schematioally in Figure 1. The "z-exis" is defined by the only

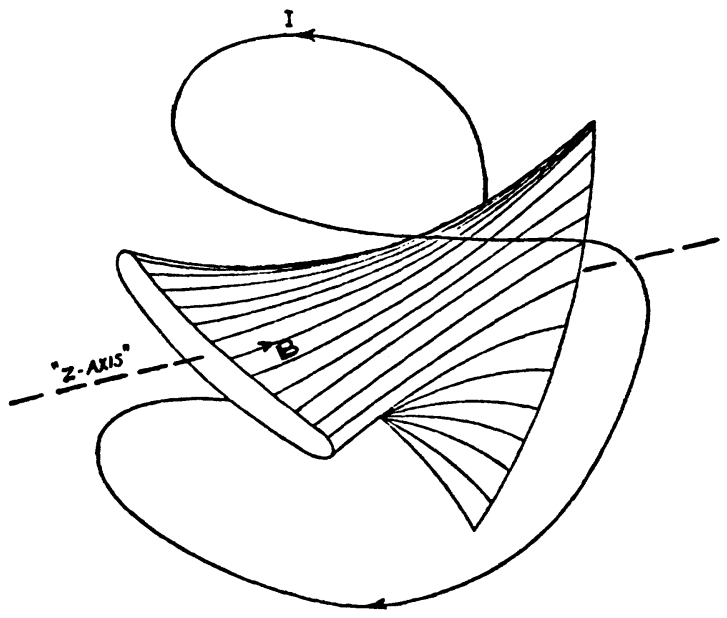

Figure 1: Current path and magnetic fleld

stralght field line that passes through the plasma. The magnetic field intensity within the plasma has its minimum value at the center of the system, and throughout the central region of the confined plasma the surfaces of constant $|B|$ are approximated by nested prolate spheroids with their axes on the z-exis.

The system is operated on a "pulsed" basis with one pulse lasting for several seconds, until

\#Work performed at Lawrence Iivermore Laboratory, University of California under the auspices of the U.S. Atomic Energy Commission. the magnet windings overheat. However, during the pulse the manet current is not completely constant and the constant $|B|$ spheroids are hence usually growing or shrinking (and appearing or disappearing at the center).

\section{The Radiometer}

A block diagram of the radiometer and a listing of some of its characteristics is given in Figure 2. The radiation is received with a cir-

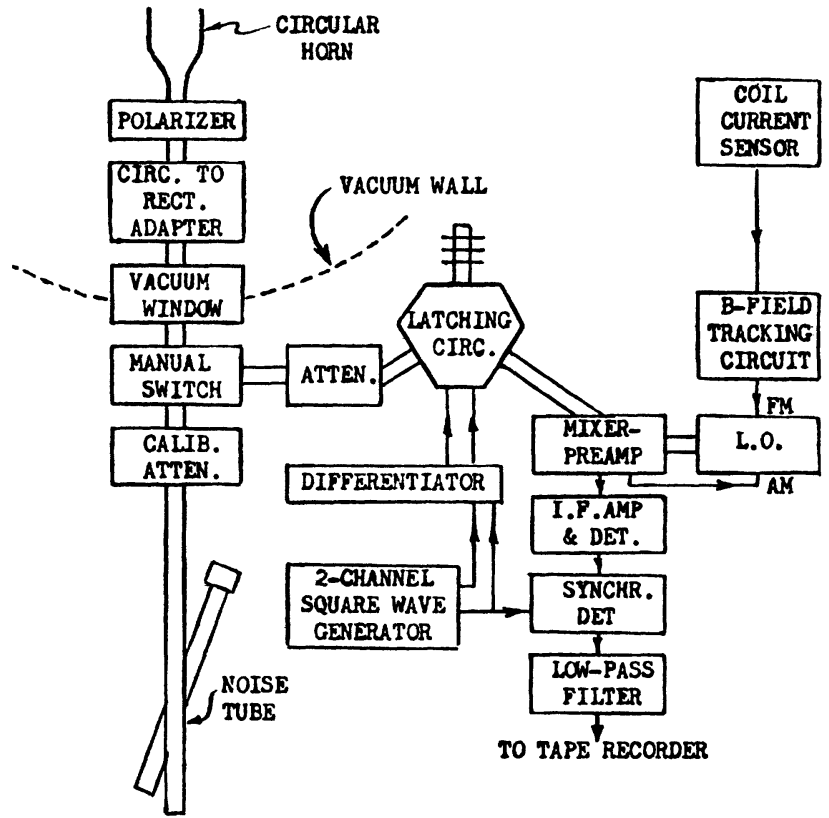

$\begin{array}{ll}\text { FREQUENCY RANGE: } & 10.2-11.6 \mathrm{cHz} \\ \text { LATCHING CIRCULATCR } & \\ \text { SWITCHING RATB } & \text { TO } 10 \mathrm{KHz} \\ \text { ISOLATION } & 20 \mathrm{DB} \\ \text { INSERTION LOSS } & 0.3 \mathrm{DB} \\ \text { I.P. CENTER FRDQ. } & 60 \mathrm{MHz} \\ \text { I.F. BANDPASS } & 40 \mathrm{MHz} \\ \text { MIXER-PREAMP N.F. } & 9.5 \mathrm{DB}\end{array}$

Flgure 2: Block diagram of the radiometer

cular horn $15 \mathrm{~cm}$ in diameter which is located on the z-axis. This direction minimizes the solid angle through which direct radiation of a given frequency is received. In this direction the radiation is circularly polarized with the same sense of rotation the electrons of the plasme experience as they spiral about the B-field lines.

The complex shape of the vacuum chamber prevents the occurrence of high-Q resonances and its volume is large enough that the average separation between resonances is about $10 \mathrm{MHz}$. To over-ride the effect of individual resonances on the coupling of horn to plasma the receiver bandwidth was chosen to be large enough ( $80 \mathrm{MHz}$ total) to 
accomodate several resonances on the average.

No attempt was made to obtain an absolute calibration of the overall sensitivity of the radiometer system. Such a calibration, properly referred to the specific volume being sampled, would of course lead to absolute values of the product $\mathrm{ne}_{\mathrm{T}}^{\mathrm{T}}$.

\section{The Polarizer}

A commercial X-band polarizer (DeMornay-Bonardi DBG-930) was used to transform the circularIf polarized radiation into linearly polarized waves. The performance of the polarizer was tested using as an analyzer a Fresnel rhomb (actually not a rhombohedron in this case). The operation of this second polarizer, first tested at optical welengths by Fresnel, is described in the optics literature.2 A principal advantage of this polarizer is that it is frequency-independent to the extent that the index of refraction is independent of frequency.

The availability of an unusually large piece of polyethylene made the construction of a microwave version of the device feasible. A sketch of a top view of this polarizer is shown in Figure 3.

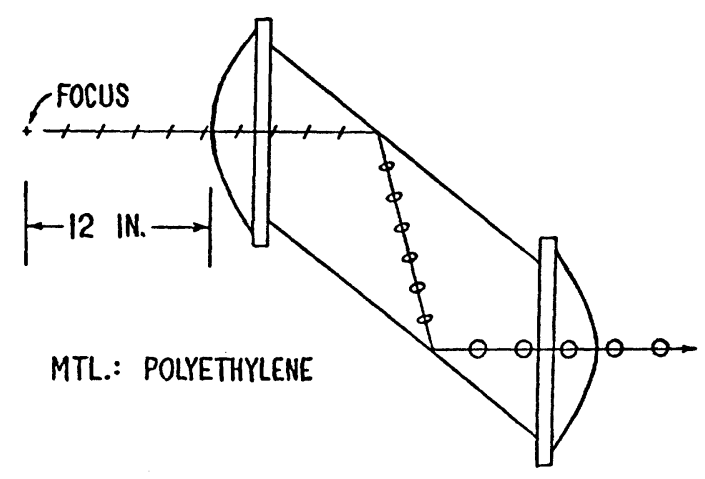

Figure 3: A microwave "Fresnel rhomb"

(By a remarkable coincidence the measured dielectric constant of the low density polyethylene at $X$-band was 2.28 , which corresponds precisely to the index of refraction, 1.51 , of the glass Fresnel used in his optical rhomb!)

The hyperbolic lenses shown in the figure give a rather large aperture angle of $43^{\circ}$ in the plane of the figure and nearly 480 perpendicular to the page in which the dimension of the prism is larger.

Since the polyethylene prism is an "all-band" microwave device, the tests shown in Figure 4 imply that its operation is at least as good as one would infer at the frequency of best discrimination between "crossed" and "uncrossed" polarizations and that the commercial unit is no worse than the overall plotted curves would indicate. The power variations with frequency in the upper curve of Figure 4 are due to the output characteristics of the unlevelled sweeper that was used for the measurement.

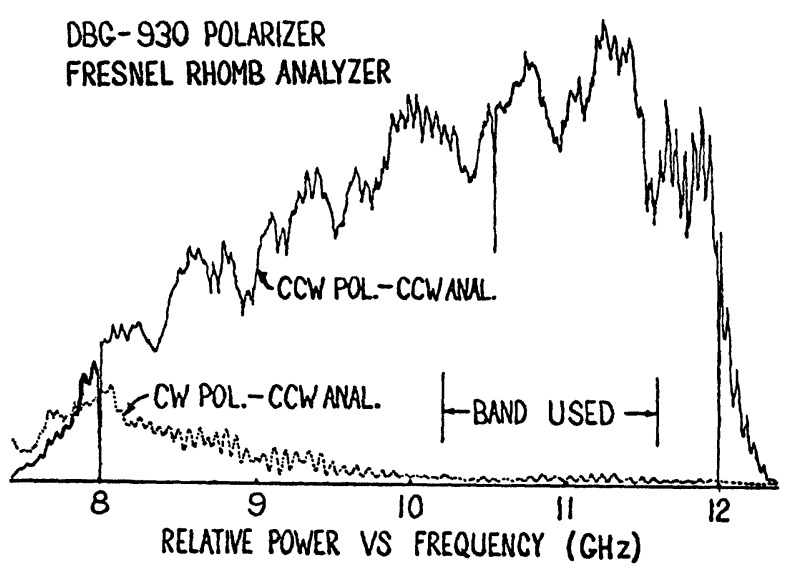

Figure 4: Test of polarizer efficiency

Sampled Region of the Plasma

The vicinity from which the sampled radiation emanates depends on the ratio of the local oscillator frequency ( $\mathrm{F}_{\mathrm{L} .0 .}$ ) to the cyclotron frequency at the center of the plasma $\left(F_{0}\right)$. For a typical ratio of 1.5 , and at an operating frequency of 10.9 $\mathrm{GHz}$, the two sidebands would receive radiation from the regions indicated in Flgure 5 if Doppler broadening were negligible. (The thickness of the lines approximately represents the

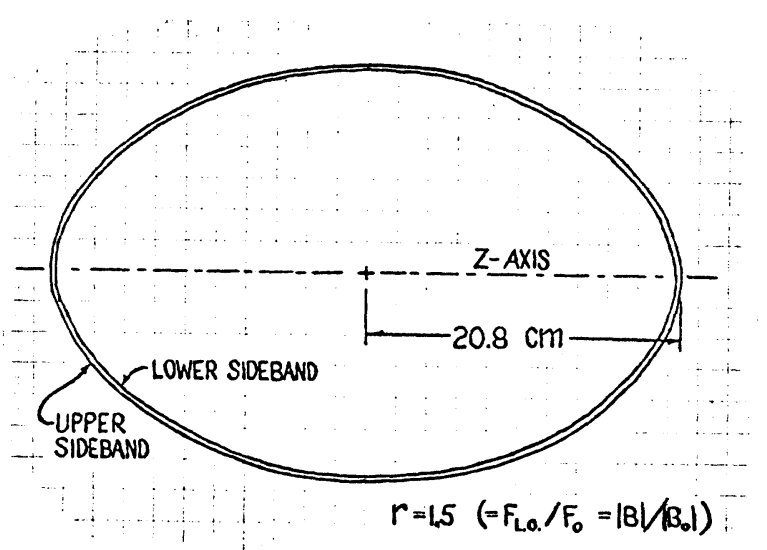

Figure 5: Plasma regions sampled by the receiver for a typical setting.

wall thickness of each spherical shell.) However, Doppler broadening of the spectrum due to the $\mathrm{z}-$ component of the electron motion is in our case of the same order of magnitude as the sideband separation with the result that each spheroidal sampling region becomes blurred until the two are essentially blended.

It is assumed that except near the center of the plasma the Doppler broadening has negligible effect on the total power received by the radiometer since the radiation that is Doppler-shifted into the accepted bands approximately equals that shifted out.

Again neglecting Doppler broadening, if the local oscillator frequency were to be swept through the spectral region of frequency $F_{0}$ a curve of the shape shown in Figure 6 would result, assuming 
uniform plasma density and temperature near the center and sharp cutoff at the edges of the receiver passmbands. In the actual case Doppler

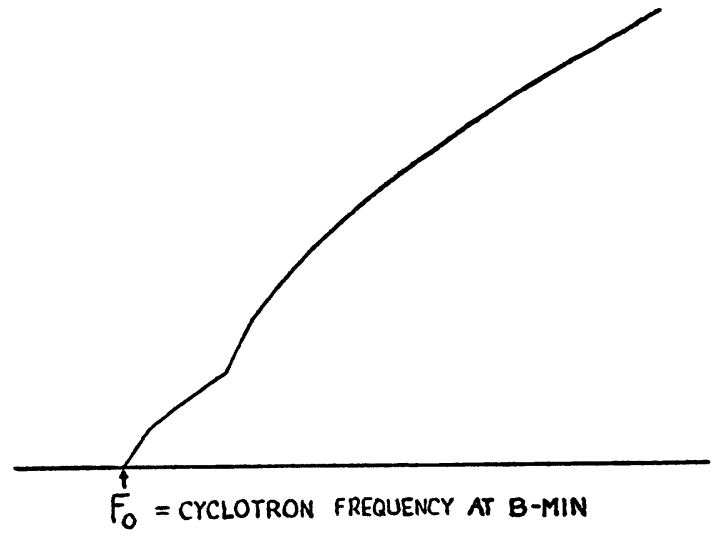

$$
\begin{gathered}
\text { POWER VS FREQUENCY } \\
\text { (NO BROADENING) }
\end{gathered}
$$

Figure 6: Idealized response as the local oscillator is swept past $\mathrm{F}_{0}$.

broadening will cause the curve to tail off into frequencies below $\mathrm{F}_{\mathrm{O}}$ and to become smoother.

Such plots were scrutinized with an aim to assessing the electron temperature but their resolution was insufficient. However, the determination of the frequency $F_{0}$ from these plots was readily made with sufficient accuracy to bring into question the measured value of the magnetic field at the center, $\left|\mathrm{B}_{0}\right|$. Recalibration of the various instruments involved resulted in agreement with the simple microwave measurement.

\section{Field-Tracking Capability}

It was noted above that the magnet current was not constant during the pulse. Thus a fixedfrequency receiver would sample radiation from changing locations within the plasma.

In order to sample radiation from a fixed, pre-selected region of the plasma the local oscillator was appropriately swept in step with the magnet current. The required tuning characteristic of the tracking circuit is plotted in Figure 7 for $r=1.5$ and also for the extreme cases of $\mathbf{r}=1.0$ (at the center of the plasma) and $\mathbf{r}=2.0$ (at the outer edges of the plasma).

The tuning range of 10.2 to $11.6 \mathrm{GHz}$ is $1 \mathrm{im}$ ited by the characteristics of the latching circulator but this proved to be adequate range to track the field in most cases.

Actually the plasma volume being sampled undergoes a small change as the local oscillator frequency is swopt but this effect has been ignored.

During sweeping of the local oscillator its power variations produce noticeable changes: in the mixer output. Rather than simply level the $\mathrm{L}_{\text {. }} 0$. output it was found to be somewhat superior to maintain the mixer crystal current at a constant value (with a feed-back loop to control the L.O. amplitude).

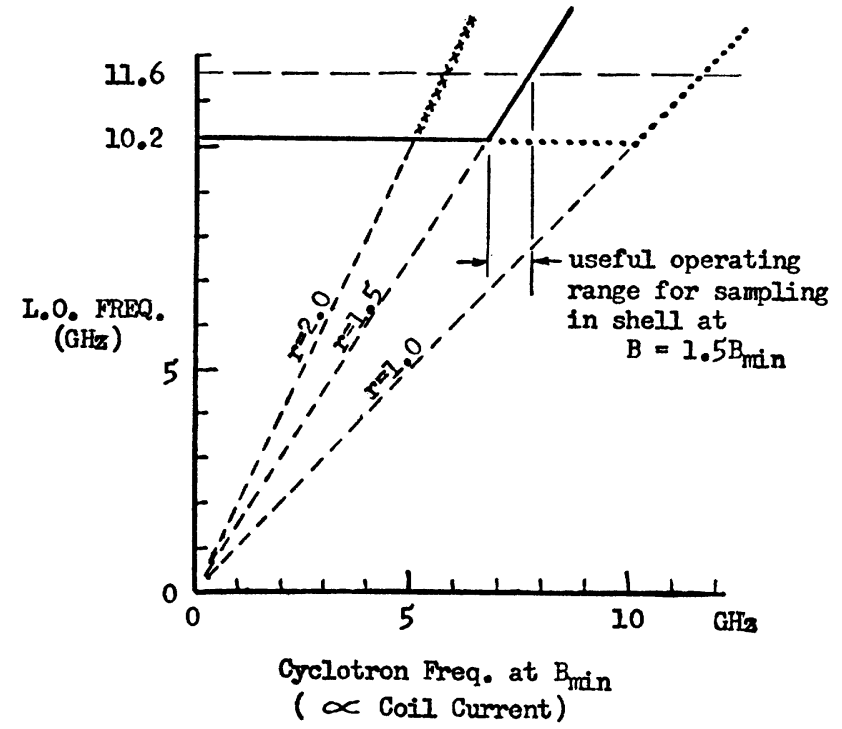

Figure 7: Tuning characteristic of the coil-current tracking circuit.

Results of the Measurements

The time-behaviour of several significant quantities during a typical pulse is shown in Figure 8.

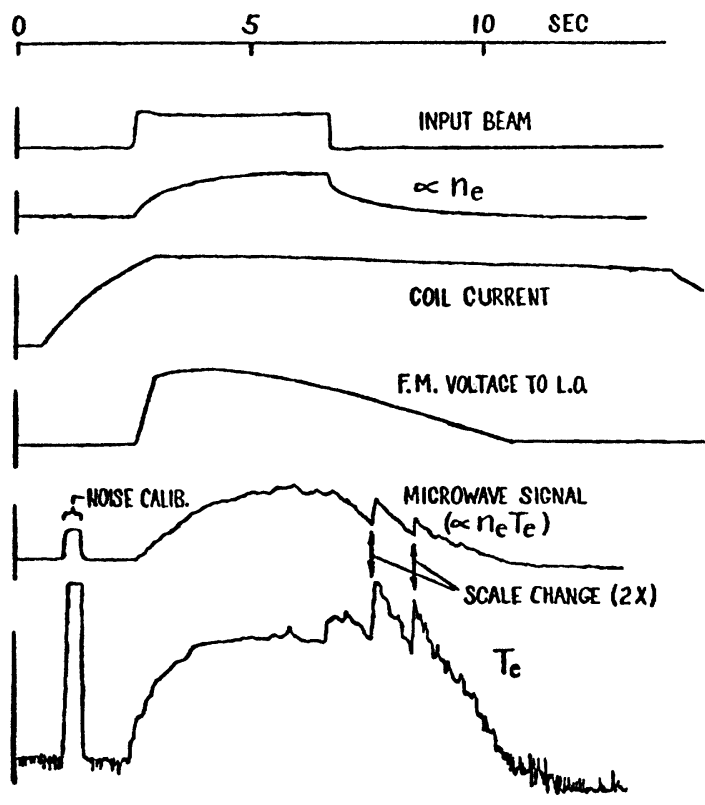

Figure 8: Measurements during a typical pulse.

The thind trace from the top (labelled " $\alpha n_{e}$ ") is derived from a detector which measures the rate of emission of fast atoms from the system which results when the energetic ions of the plasma undergo charge exchange collisions with the neutral atoms of the background gas. For a particular pulse this signal is proportional to the electron density, $n_{e}$, if one assumes quasi-neutrality of the plasma.

The presence of a non-saturating and non-zero 
"F.M. voltage to L.0." (on the 5th trace) indicates that the tracking circuit is operating in the desired range.

Although the microwave signal amplitude has no absolute calibration, its pulse-to-pulse comparison is made by the use of a reference signal from the noise source. (In this particular case the calibrating pulse from the noise tube was applied through an attenuation of $12 \mathrm{db}$.

The bottom trace, obtained by dividing the microwave signal by the fast atom signal, is thus a measure of relative electron temperature. The pronounced increase in this temperature upon cessation of the input beam is rather dramatic even though it is expected.

A discussion of the measurements and their theoretical implications in perspective with other measurements of the plasma has been given by Futch et 21.3 One of the theoretical predictions is that the electron cyclotron radiation should vary as $\left(n_{i}\right) 1.4$. The data of Figure 9 (taken from Ref. 3) give strong support for this prediction.

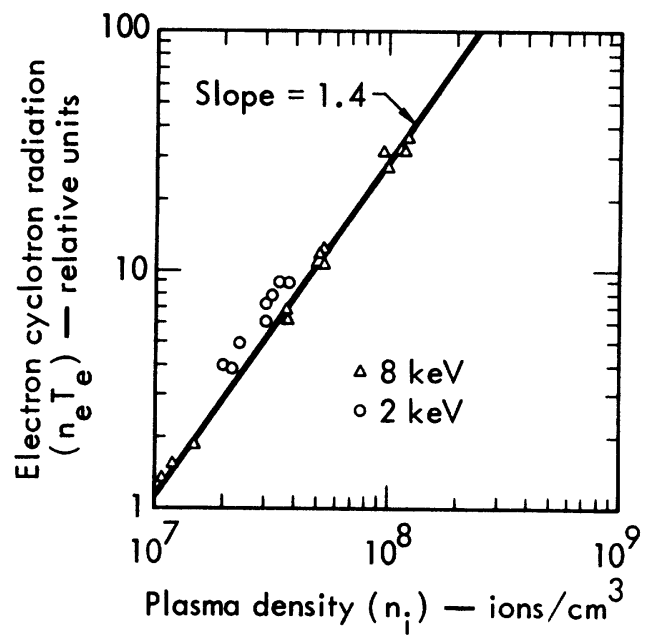

Figure 9: Intensity of radiation emitted at the electron cyclotron frequency as a function of $n_{j}$, for hot-ion energies of $8 \mathrm{keV}$ and $2 \mathrm{keV}$.

\section{Possible Future Measurements}

1. Although an absolute determination of the emitted radiation may be difficult an attempt to make this measurement seems to be warranted.

2. Some limited tests on the Alice system have employed low-level electron cyclotron heating pulses in conjunction with the observation of cyclotron emission. This makes the communication with the electrons a bilateral process. Since both processes have the same spatial resolution capabilities their combination might be particularly useful in some circumstances.

3. With the advent of superconducting magnets the nature of some problems of the radiometer will change. The longer integration times available will permit greater sensitivity and resolution and the requirement for tracking the fleld will vanish.
4. Consideration is being given to the possibility of determining the electron temperature by measuring the ratio of emitted radiation at the cyclotron frequency and at its second harmonic.

\section{Acknowledgements}

A. H. Futch provided the chief motivation for these measurements and participated fully in most of the aspects of this work. The author also acknowledges the encouragement and assistance given by C. C. Damm, R. F. Post, J. H. Foote, K.G. Moses and the entire staff of the Alice project.

\section{$\underline{\text { References }}$}

1. C. C. Damm et al., in Proceedings of a Conference on Plasma Physics and Controlled Nuclear Fusion Research, Novosibirsk, U.S.S.R., 1968 (Intermational Atomic Energy Agency, Vienna, Austria, 1969) Vol. II, p. 253.

2. For example see: F. A. Jenkins and H. E. White, Fundamentals of Physical Optics, McGraw-Hill Book Co., Inc., New York (1937) p. 396.

3. A. H. Futch, C. C. Damm, J. H. Foote, A. I. Gardner and J. K. Killeen, Phys. Fluids 14, 1542 (1971). 